\title{
Inactivation of AMPK Mediates High Phosphate-Induced Extracellular Matrix Accumulation via NOX4/TGFB-1 Signaling in Human Mesangial Cells
}

\author{
Alexandros Papadimitriou ${ }^{a} \quad$ Elisa B.M.I. Peixoto ${ }^{a} \quad$ Kamila C. Silva ${ }^{a}$ \\ Jacqueline M. Lopes de Faria ${ }^{a}$ José B. Lopes de Faria ${ }^{a}$
}

aRenal Pathophysiology Laboratory, Investigation on Diabetes Complications, Faculty of Medical Sciences, State University of Campinas (Unicamp), Campinas, São Paulo, Brazil

\section{Key Words}

AMPK • Extracellular matrix accumulation $\bullet$ Mesangial cells $\bullet$ NOX4 $\bullet$ Phosphate $\bullet$ Transforming growth factor $B-1$

\begin{abstract}
Background/Aims: High phosphate $(\mathrm{Pi})$ levels and extracellular matrix (ECM) accumulation are associated with chronic kidney disease progression. However, how high Pi levels contribute to ECM accumulation in mesangial cells is unknown. The present study investigated the role and mechanism of high Pi levels in ECM accumulation in immortalized human mesangial cells (iHMCs). Methods: iHMCs were exposed to normal $(0.9 \mathrm{mM})$ or increasing Pi concentrations (2.5 and $5 \mathrm{mM}$ ) with or without diferent blockers or activators. NOX4, phosphorylated AMPK (p-AMPK), phosphorylated SMAD3 (p-SMAD3), fibronectin ( $F / N)$, collagen IV (CIV) and alpha-smooth muscle actin ( $\alpha-S M A)$ expression was assessed via western blot and immunofluorescence. Lucigenin-enhanced chemiluminescence, and dihydroethidium (DHE) assessed NADPH oxidase activity and superoxide (SO), respectively. Results: In iHMCs, a Pi transporter blocker (PFA) abrogated high Pi-induced AMPK inactivation, increase in NADPH oxidase-induced reactive oxygen species (ROS) levels, NOX4, p-SMAD3, $\alpha$-SMA and C-IV expression. AMPK activation by AICAR, NOX4 silencing or NADPH oxidase blocker prevented high Pi-induced DHE levels, p-SMAD3, F/N, C-IV and $\alpha$-SMA expression. Conclusion: AMPK inactivation with NOX4-induced ROS formation and transforming growth factor B-1 (TGFB1) signaling activation mediates high Pi-induced ECM accumulation in iHMCs. Maneuvers increasing AMPK or reducing NOX4 activity may contribute to renal protection under hyperphosphatemia.




\section{Introduction}

Clinical and experimental data indicate that elevated plasma phosphate (Pi) may contribute to progression of chronic kidney disease (CKD) [1-5] by a not fully elucidated mechanism. In spite of that, the effect of high Pi levels per se on kidney cells may play a role, since it has been shown that addition of higher Pi concentrations to the culture medium of kidney fibroblasts elicits increased extracellular matrix (ECM) production [6]. ECM accumulation in the glomeruli and tubulointerstitial area are well recognized factors in the development of glomerulosclerosis and interstitial fibrosis, both anatomical markers of renal disease progression [7, 8]. Glomerular mesangial cells exposed to stimuli that are known to contribute to renal disease progression, such as high glucose, angiotensin II, and transforming growth factor (TGFß)-1, produce increased amounts of ECM [9-11]. Whether high Pi promotes ECM accumulation in mesangial cells has not yet been investigated.

As a serine/threonine kinase, 5' adenosine monophosphate-activated protein kinase (AMPK) is an important metabolic sensor expressed ubiquitously in almost all eukaryotic cells [12]. The activity and subunit composition of AMPK are expressed in a cell- and tissue-specific manner, with the $\alpha_{1}$ - and $\alpha_{2}$-subunits expressed in the kidney and in glomerular mesangial cells [13]. The activation of AMPK requires the phosphorylation of a critical threonine residue $\left(\mathrm{Thr}^{172}\right)$ in the activation loop of the $\alpha$-subunit [14]. It has been previously established that AMPK is involved in regulating many cellular functions, including endothelial nitric oxide synthase activation, angiogenesis, and mitochondrial function [15]. Further, AMPK may also be involved in the pathogenesis of kidney fibrosis [16]. To this end, it has been demonstrated that AMPK activation by AICAR prevents renal fibrosis in the rat model of renal ablation [17], and also in experimental diabetic nephropathy $[18,19]$. We have recently demonstrated that human mesangial cells exposed to high glucose concentrations display ECM accumulation mediated by reduction in AMPK followed by an activation of NOX4 and TGFß-1 [11]. Also, oxidative stress, induced by NOX4 activation, contributes to ECM accumulation, which has been demonstrated in mesangial cells treated with angiotensin II or high glucose levels [9, $10,20]$. The effect of high phosphate levels on AMPK, NOX4, and TGF beta in mesangial cells has not been investigated.

The aim of the present study was to investigate whether human mesangial cells exposed to high Pi concentrations display increased ECM accumulation mediated by AMPK/NOX4/ TGFß-1 signaling.

\section{Materials and Methods}

\section{Reagents}

All reagents were purchased from Sigma (St. Louis, MO) unless otherwise stated.

\section{Immortalized human mesangial cell (iHMC) culture}

Dr. Nestor Schor (Department of Medicine, Nephrology Division, Federal University of São Paulo, Brazil) kindly provided the iHMCs. They were originally from Dr. Bernhard Banas (Nephrology Center, Medical Policlinic, Ludwig-Maximilian University of Munich, Germany) and were cultured as described in previous studies $[21,22]$. The concentrations of treatments used in high Pi $(2.5$ and $5 \mathrm{mM})$ medium in all experiments were chosen after carrying out a thiazolyl blue tetrazolium bromide (MTT) assay (data not shown).

\section{Experimental conditions of iHMCs}

The iHMCs were kept for $24 \mathrm{~h}$ without serum in Dulbecco's modified Eagle's medium (DMEM) supplemented with $10 \%$ fetal calf serum (Life Technologies, Grand Island, NY) containing $0.9 \mathrm{mM} \mathrm{Pi}$ (control) or appropriate increasing amounts of sodium phosphate buffer $\left(1 \mathrm{M} \mathrm{Na}_{2} \mathrm{HPO} / \mathrm{NaH}_{2} \mathrm{PO}\right.$, $\left.\mathrm{pH} 7.4\right)$ to achieve final Pi concentrations of 2.5 and $5 \mathrm{mM}$. The iHMCs were also treated with 2.5 and $5 \mathrm{mM}$ Pi in the presence of phosphonoformic acid (PFA, $1 \mathrm{mM}$ ) as a specific inhibitor of the sodium-phosphate transporter that 
Papadimitriou et al.: High Phosphate Levels Increase ECM Accumulation via AMPK Inactivation in Mesangial Cells

Fig. 1. High phosphate levels by sodium-phosphate transporter increase ECM accumulation via activation of TGFß-1 signaling. Representative western blot analysis of collagen IV (a), alpha-smooth muscle actin ( $\alpha$-SMA) (c) and phosphorylated SMAD3 (e) expression in iHMCs under $0.9 \mathrm{mM}$ or $2.5 \mathrm{mM}$ or $5 \mathrm{mM}$ phosphate alone or in combination with PFA (1 mM) followed by quantification of collagen IV/actin (b), $\alpha$-SMA/ actin (d) and pSMAD3/TSMAD3/actin (f) ratios. The bars represent mean \pm SEM of band intensities (arbitrary densitometric units) of 3 independent experiments and $\mathrm{n}=5$ for each treatment. ${ }^{*} \mathrm{p}=$ 0.01 and $\# p<0.0001$ vs. 0.9 $\mathrm{mM}$ phosphate for (b). ${ }^{*} \mathrm{p}<$ 0.0001 vs. $0.9 \mathrm{mM}$ phosphate for (d). ${ }^{*} \mathrm{p}=0.012$ and $\# \mathrm{p}<$ 0.0001 vs. $0.9 \mathrm{mM}$ phosphate for (f).

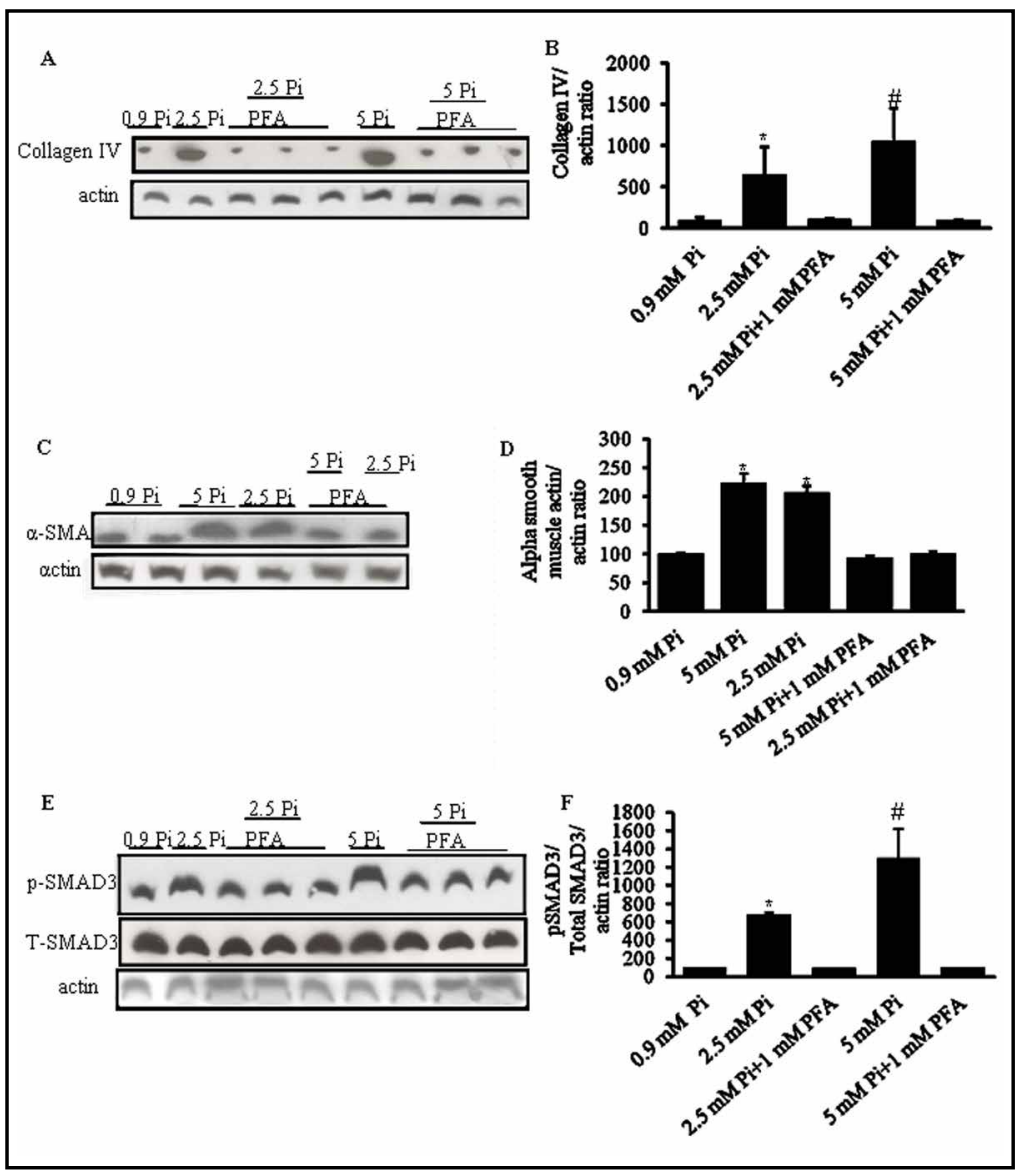

blocks phosphate transport into the cells, or in diphenyleneiodonium as an NADPH oxidase blocker (DPI, 50 $\mathrm{nM})$, or in small interfering RNA (siRNA) or scramble (Src) for NOX4 (200 nM), or in 5-aminoimidazole-4carboxamide ribonucleotide (AICAR, $1 \mathrm{mM}$ ) as an activator of AMPK or in mannitol ( $30 \mathrm{mM}$ ) as an osmotic control.

\section{Transient transfection with siRNAs}

The siRNA duplexes and scrambled siRNA corresponding to human NOX4 were obtained from Invitrogen (Carlsbad, CA). The transient transfection of siRNAs was carried out using lipofectamine transfection reagent (Invitrogen, Carlsbad, CA). The siRNA and scrambled (200 nM) for human NOX4 (Invitrogen, Carlsbad, CA) were formulated with lipofectamine transfection reagent according to the manufacturer's instructions after incubation for $24 \mathrm{~h}$ in the absence of serum and antibiotics [20].

\section{NADPH oxidase activity}

The NADPH oxidase activity was measured by the lucigenin-enhanced chemiluminescence method [23].

\section{Dihydroethidium (DHE) measurement of superoxide production}

Superoxide (SO) production was indicated with DHE. Qualitative assessement of SO was carried out in the iHMCs after they were kept for $24 \mathrm{~h}$ with $0.9,2.5$ or $5 \mathrm{mM} \mathrm{Pi}$ in the presence of AICAR, followed by incubation with $10 \mu \mathrm{M}$ DHE fluorescent probes at $37^{\circ} \mathrm{C}$ for $30 \mathrm{~min}$. Images were then taken with an Olympus FluoView fluorescent microscope (Olympus Corporation of the Americas, Melvile, NY). To quantify SO levels, the same procedure used for the qualitative analysis of SO was applied. Relative fluorescence was measured using a fluorescence plate reader (SynergyMx; Biotek) at excitation and emission wavelengths of 520 and $610 \mathrm{~nm}$, respectively. The relative fluorescence values were corrected by the number of cells in each treatment. 
Fig. 2. High phosphate levels inactivate AMPK and increase NADPH oxidase activity and NOX4 expression by sodium-phosphate transporter in iHMCs. Representative western blot analysis of phosphorylated AMPK (pAMPK) $\quad\left(\mathrm{Thr}^{172}\right) \quad$ (a) expression in iHMCs under $0.9 \mathrm{mM}$ or 2.5 $\mathrm{mM}$ or $5 \mathrm{mM}$ phosphate alone or in combination with PFA (1 mM) followed by quantification of pAMPK/TAMPK/actin (b), ratio. The bars represent mean \pm SEM of band intensities (arbitrary densitometric units) of 3 independent experiments and $\mathrm{n}=5$ for each treatment. ${ }^{*} \mathrm{p}$ $=0.02$ and $\# \mathrm{p}<0.0001$ vs. $0.9 \mathrm{mM}$ phosphate. (c). NADPH-dependent ROS generation in iHMCs in conditions as indicated, and expressed as a percentage of rela-

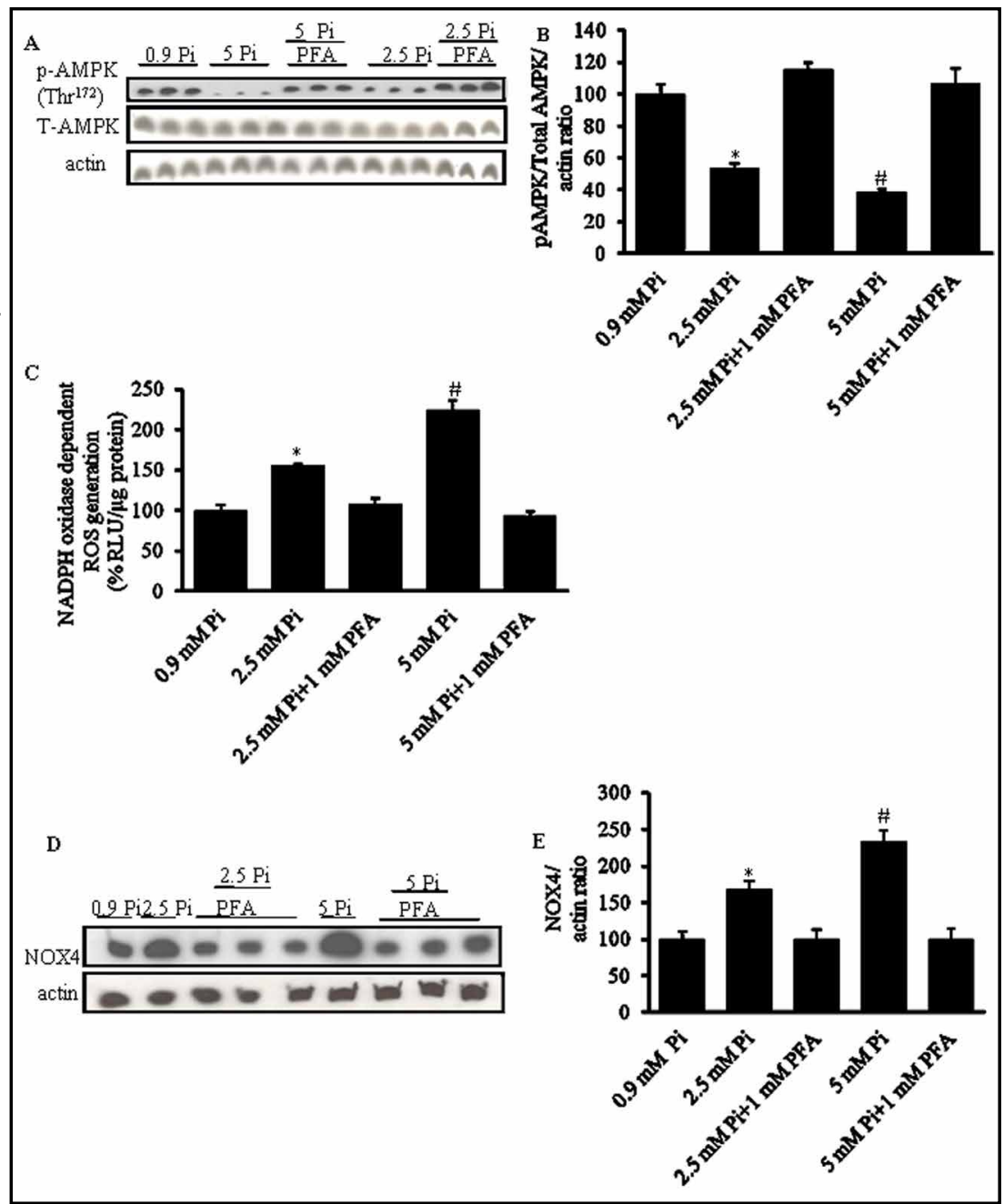
tive luminescence units (RLU)/ $\mu$ g protein. The bars represent mean $\pm \mathrm{SD}$ of 3 independent experiments and $\mathrm{n}=6$ for each treatment. ${ }^{*} \mathrm{p}=0.01$ and $\# \mathrm{p}<0.0001$ vs. $0.9 \mathrm{mM}$ phosphate. (d). Representative western blot analysis of NOX4 expression in iHMCs in conditions as indicated followed by quantification of NOX4/actin (e) ratio. The bars represent mean \pm SEM of band intensities (arbitrary densitometric units) of 3 independent experiments and $\mathrm{n}=5$ for each treatment. ${ }^{*} \mathrm{p}=0.02$ and $\# \mathrm{p}<0.0001$ vs. $0.9 \mathrm{mM}$ phosphate.

\section{Western blot analysis}

The samples and Western blots were prepared as described in previous studies [23-25]. The following primary antibodies were used: goat anti-type IV collagen (1:500, Southern Biotech, Birmingham, AL), goat anti-fibronectin (F/N) (1:500, EMD Millipore, Billerica, MA), rabbit anti-NOX4 (1:500, Santa Cruz Biotechnology, Danvers, MA), rabbit anti-alpha smooth muscle actin ( $\alpha$-SMA) (1:500, Abcam, Cambridge, MA), rabbit phosphorylated AMPK $\left(\mathrm{Thr}^{172}\right)$ and LKB1 $\left(\mathrm{Ser}^{428}\right)$ (1:1000, Cell Signalling Technology, Danvers, MA), rabbit total AMPK and LKB1 (1:1000, Cell Signalling Technology), rabbit phosphorylated SMAD3 (1:500, Cell Signalling Technology, Danvers, MA), and total rabbit SMAD3 (1:1000, Cell Signalling Technology, Danvers, MA). To verify the uniformity of the protein load and transfer efficiency across the test samples, the membranes were reprobed with actin (goat polyclonal anti-actin antibody, diluted 1:1000; Santa Cruz Biotechnology, Danvers, MA).

\section{Immunofluorescence for $\alpha$-SMA}

Immunofluorescence staining was performed by incubating fixed iHMCs in $4 \%$ paraformaldehyde with $\alpha$-SMA primary antibody (1:10; Abcam) and rhodamine-conjugated secondary antibody (1:500, BD 
Fig. 3. AICAR prevents high phosphate-induced AMPK inactivation in iHMCs. Representative western blot analysis of phosphorylated AMPK (pAMPK) (Thr ${ }^{172}$ ) (a and c) in iHMCs under $0.9 \mathrm{mM}$ phosphate or $2.5 \mathrm{mM}$ or $5 \mathrm{mM}$ phosphate alone or in combination with AICAR (1 mM) followed by quantification of pAMPK/TAMPK/actin ratio ( $b$ and $d$ ). The bars represent mean \pm SEM of band intensities (arbitrary densitometric units) of 3 independent experiments and $\mathrm{n}=5$ for each treatment. ${ }^{* *} \mathrm{p}=$ 0.01 vs. $0.9 \mathrm{mM}$ phosphate for (a). ${ }^{*} \mathrm{p}=0.04$ vs. $0.9 \mathrm{mM}$ phosphate for (b).

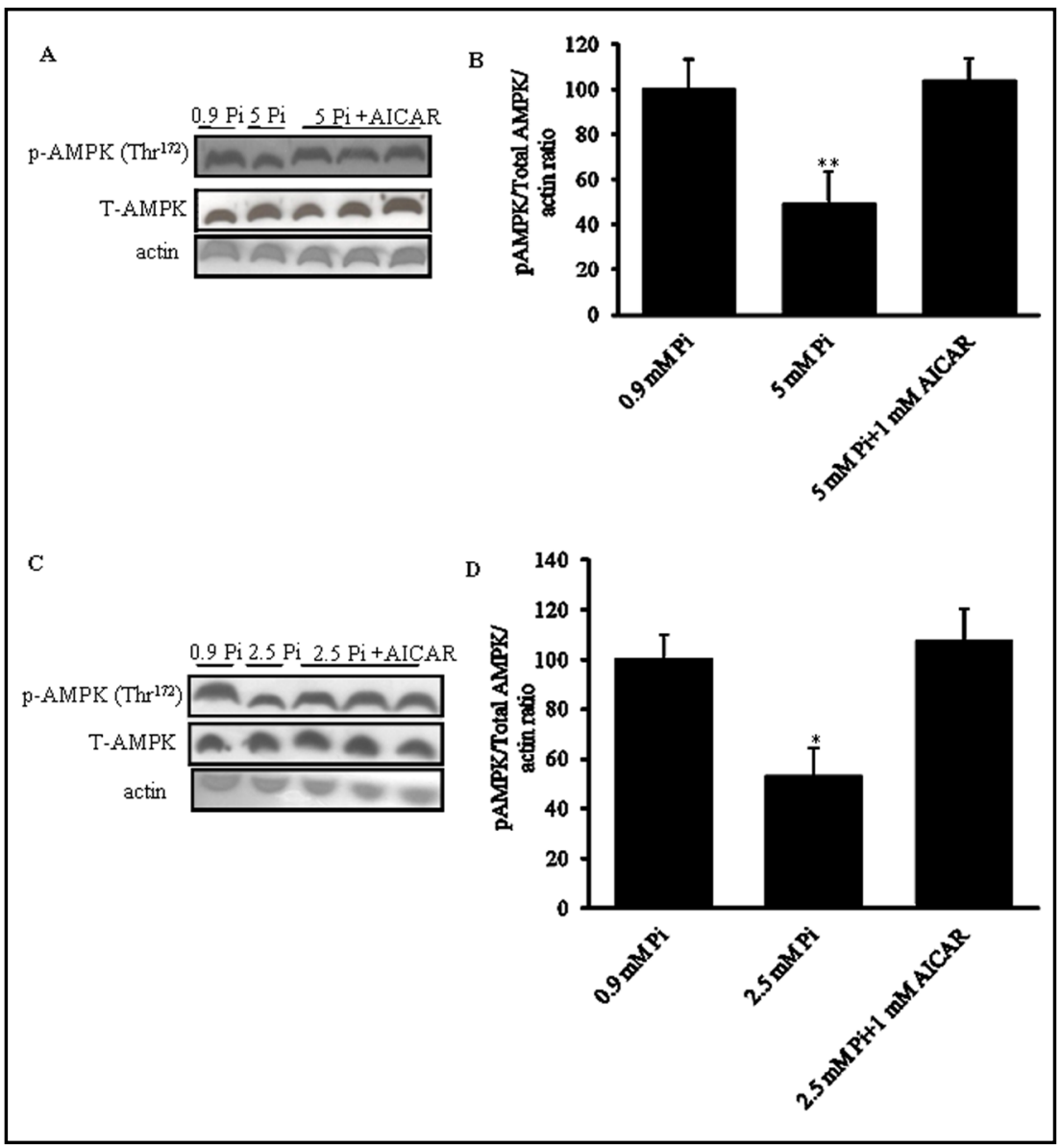

Transduction Labs, San Jose, CA). Images were then taken and analyzed by Confocal Microscopy (Leica TCS SP5 II), 40x of magnification.

\section{Statistical analysis}

Results are expressed as mean \pm standard error of mean (SEM), and comparisons between multiple groups are performed with one-way analysis of variance (ANOVA) followed by the Bonferroni test, while a t-test was used for comparisons between two groups. Analyses are considered significant at $\mathrm{p}<0.05$. The analyses were performed using StatView software (SAS Institute Inc., Cary, NC).

\section{Results}

High phosphate levels increased ECM accumulation associated with elevated TGF $\beta-1$ signaling

The expression levels of both collagen IV, $\alpha$-SMA, and phosphorylated SMAD3 were significantly elevated in iHMCs exposed to high Pi levels (Fig. 1a-f) and they were both prevented after PFA treatment.

Signaling pathways induced by phosphate in mesangial cells

High phosphate levels induced oxidative stress by NADPH oxidase activation via NOX4 following inactivation of AMPK. The expression levels of phosphorylated AMPK were significantly reduced (Fig. 2a and b), and ROS formation via NADPH oxidase (Fig. 2c), and NOX4 expression (Fig. 2d and e) increased in a concentration-dependent manner in iHMCs exposed to high Pi levels, and these were reversed by PFA treatment. Treatment of mesangial cells with AICAR, an AMPK activator (Fig. 3a-d), prevented the increment in NOX4 expression 
Cellular Physiology and Biochemistry
Cell Physiol Biochem 2014;34:1260-1272

\begin{tabular}{l|l}
\hline DOI: $10.1159 / 000366336$ & C 2014 S. Karger AG, Basel
\end{tabular}

Publisnea ontme. september 29, $2014 \quad$ www.karger.com/cpb

Papadimitriou et al.: High Phosphate Levels Increase ECM Accumulation via AMPK Inactivation in Mesangial Cells
Fig. 4. High phosphate levels increase NADPH oxidase activation and particularly NOX4 expression via AMPK inactivation in iHMCs. Representative western blot analysis of NOX4 (a) in iHMCs under $0.9 \mathrm{mM}$ or $2.5 \mathrm{mM}$ or $5 \mathrm{mM}$ phosphate alone or in combination with AICAR (1 mM) followed by quantification of NOX4/actin (b) ratio. The bars represent mean \pm SEM of band intensities (arbitrary densitometric units) of 3 independent experiments. ${ }^{*} \mathrm{p}=0.035$ and $\# \mathrm{p}$ $<0.0001$ vs. $0.9 \mathrm{mM}$ phosphate. (c). NADPH-dependent ROS generation in iHMCs in conditions as indicated and expressed as percentage of relative luminescence units (RLU)/ $\mu$ g protein. The bars represent mean \pm SD of 3 independent experiments and $\mathrm{n}=5$ for each treatment. ${ }^{*} \mathrm{p}=$ 0.01 and $\# p<0.0001$ vs. $0.9 \mathrm{mM}$ phosphate.

Fig. 5. High phosphate levels increase SO production via AMPK inactivation in iHMCs. Qualitative assessment of SO formation (a). SO levels were assessed by incubating iHMCs with $10 \mu \mathrm{M}$ DHE for 30 min. Images were then taken under fluorescent microscope and colored images are illustrating SO signal for each treatment (a). Quantitative assessment of SO formation via fluorometric measurement (b). The bars represent mean \pm SEM of 3 independent experiments and values are expressed as percentage of fluorescence units. ${ }^{*} \mathrm{p}<0.001$ vs. $0.9 \mathrm{mM}$ phosphate.
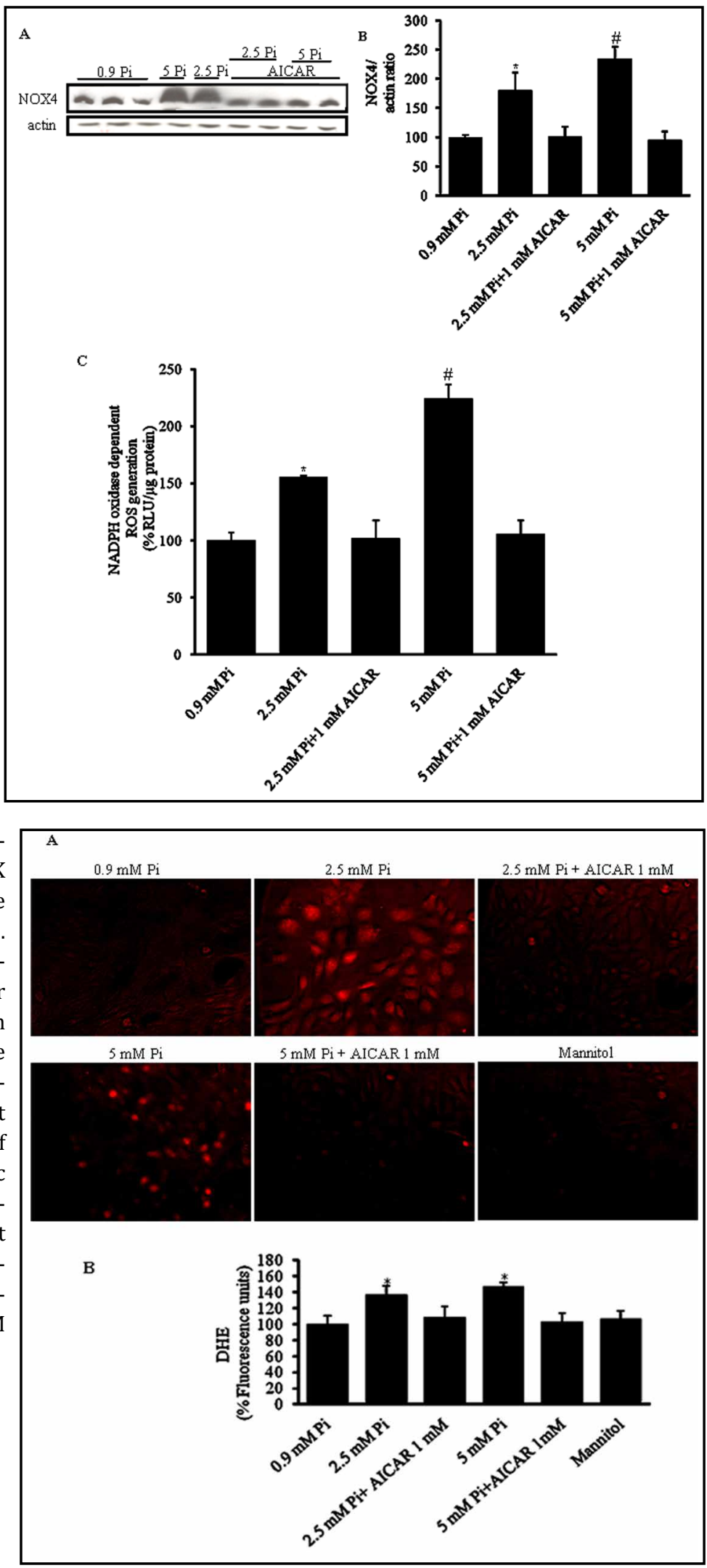

(Fig. 4a and b), NADPH oxidase activity (Fig. 4c), SO production (Fig. 5a-b), phosphorylated SMAD3 (Fig. 6a and b), collagen IV (Fig. 6c and d), fibronectin (Fig. 6e and f) and $\alpha$-SMA (Fig. 
Fig. 6. High phosphate levels increase TGFß-1 signaling and ECM accumulation following AMPK inactivation in iHMCs. Representative western blot analysis of phosphorylated SMAD3 (a), collagen IV (c) and fibronectin (e) expression in iHMCs under $0.9 \mathrm{mM}$ or 2.5 $\mathrm{mM}$ or $5 \mathrm{mM}$ phosphate alone or in combination with AICAR (1 mM) followed by quantification of pSMAD3/TSMAD3/ actin (b), collagen IV/actin (d) and fibronectin/ actin (f) ratios. The bars represent mean \pm SEM of band intensities (arbitrary densitometric units) of 3 independent experiments and $\mathrm{n}=5$ for each treatment. ${ }^{*} \mathrm{p}=0.03$ and $\# \mathrm{p}<0.0001$ vs. $0.9 \mathrm{mM}$ phosphate for (b). ${ }^{*} \mathrm{p}=$ 0.01 and $\# \mathrm{p}<0.0001$ vs. $0.9 \mathrm{mM}$ phosphate for (d). . ${ }^{*}$ p $<0.0001$ vs. 0.9 $\mathrm{mM}$ phosphate for (f).

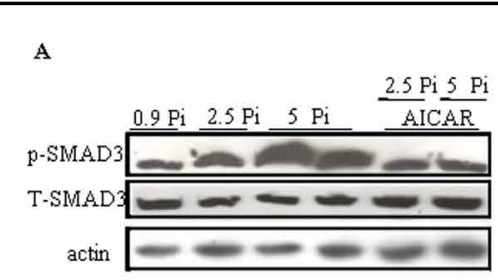

$\mathrm{B}$
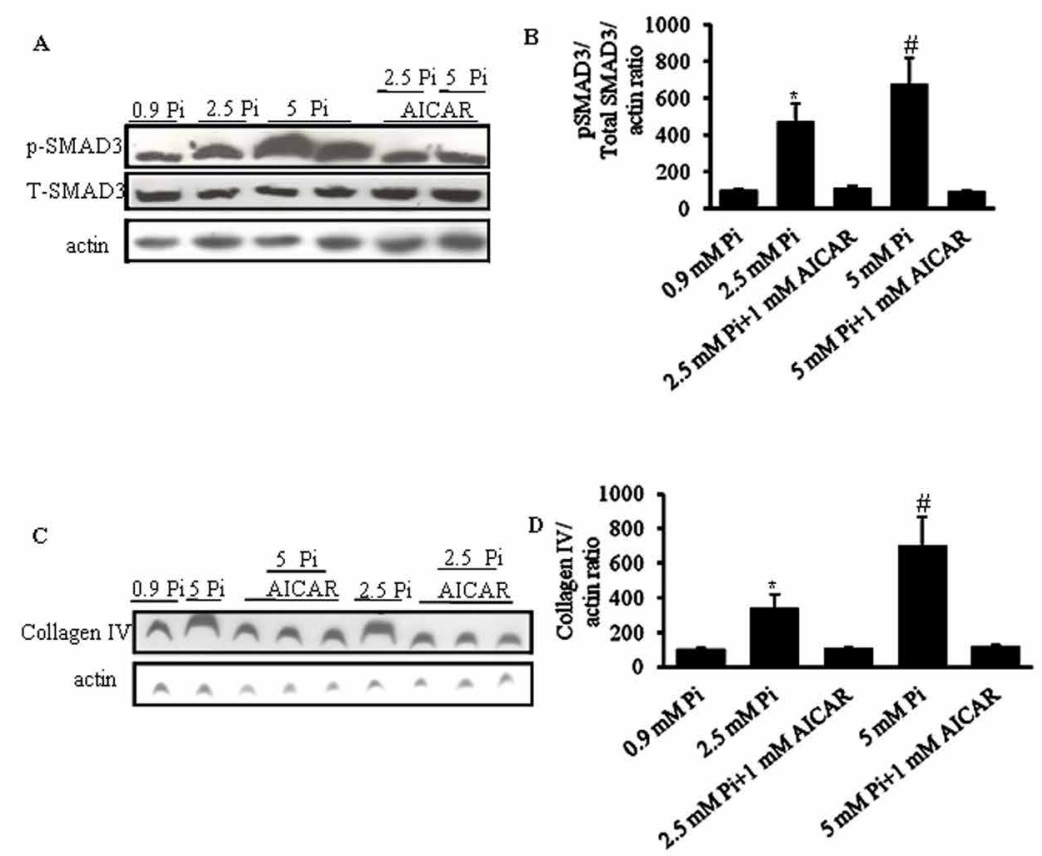

E
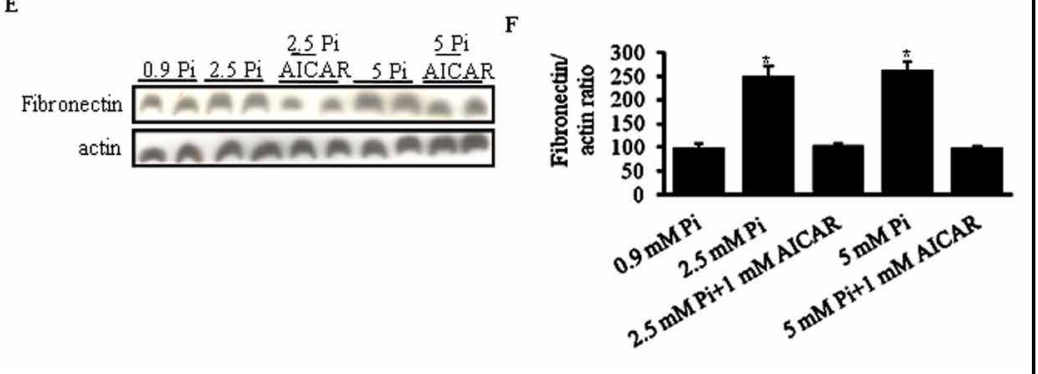

7a-c) expressions induced by high Pi levels. Inactivation of AMPK by high Pi levels may occur due to reduction of phosphorylated liver kinase B1 (LKB1), a critical upstream activator of AMPK (Fig. 8a and b).

Activation of NADPH oxidase and particularly NOX4 by high phosphate levels increases TGFß-1 signaling and ECM accumulation. Pharmacological blockade of NADPH oxidase with DPI (Fig. 9a-d) or NOX4 silencing (Fig. 10a-d) abrogated high Pi-induced increase in phosphorylated SMAD3 (Fig. 9a-b and Fig. 10e-f), collagen IV (Fig. 9c-d and Fig. 11a-b) and fibronectin (Fig. 11c-d) expression.

\section{Discussion}

Thus far, the cellular mechanism by which hyperphosphatemia is involved in renal disease progression has not been assessed in great detail. In the current study, we showed, for the first time, that in human mesangial cells, elevated Pi medium concentrations via the sodium-phosphate transporter lead to an increase in the pro-fibrotic proteins, collagen IV and fibronectin. Elevated Pi also activates mesangial as showed by increased $\alpha$-SAM actin expression. High Pi levels inactivate AMPK, leading to the upregulation of NOX4 expression and subsequent activation of TGFß-1 signaling via phosphorylation of SMAD3 that ultimately leads to increased collagen IV, fibronectin and $\alpha$-SMA levels (Fig. 12). 
Papadimitriou et al.: High Phosphate Levels Increase ECM Accumulation via AMPK Inactivation in Mesangial Cells

Fig. 7. High phosphate levels increase alphasmooth muscle actin ( $\alpha$-SMA) following AMPK inactivation in iHMCs. Representative western blot analysis of $\alpha$-SMA expression (a) in iHMCs under $0.9 \mathrm{mM}$ or 2.5 $\mathrm{mM}$ or $5 \mathrm{mM}$ phosphate alone or in combination with AICAR (1 mM) followed by quantification of $\alpha$-SMA/actin ratio (b). The bars represent mean \pm SEM of band intensities (arbitrary densitometric units) of 3 independent experiments and $\mathrm{n}=5$ for each treatment. ${ }^{*} \mathrm{p}<$ 0.0001 vs. $0.9 \mathrm{mM}$ phosphate (c). Immunofluorescence staining for alpha-smooth muscle actin ( $\alpha$-SMA) in iHMCs treated as indicated. TOPRO marker shows nuclear staining. Images were analyzed by Confocal Microscopy, 40x of magnification.

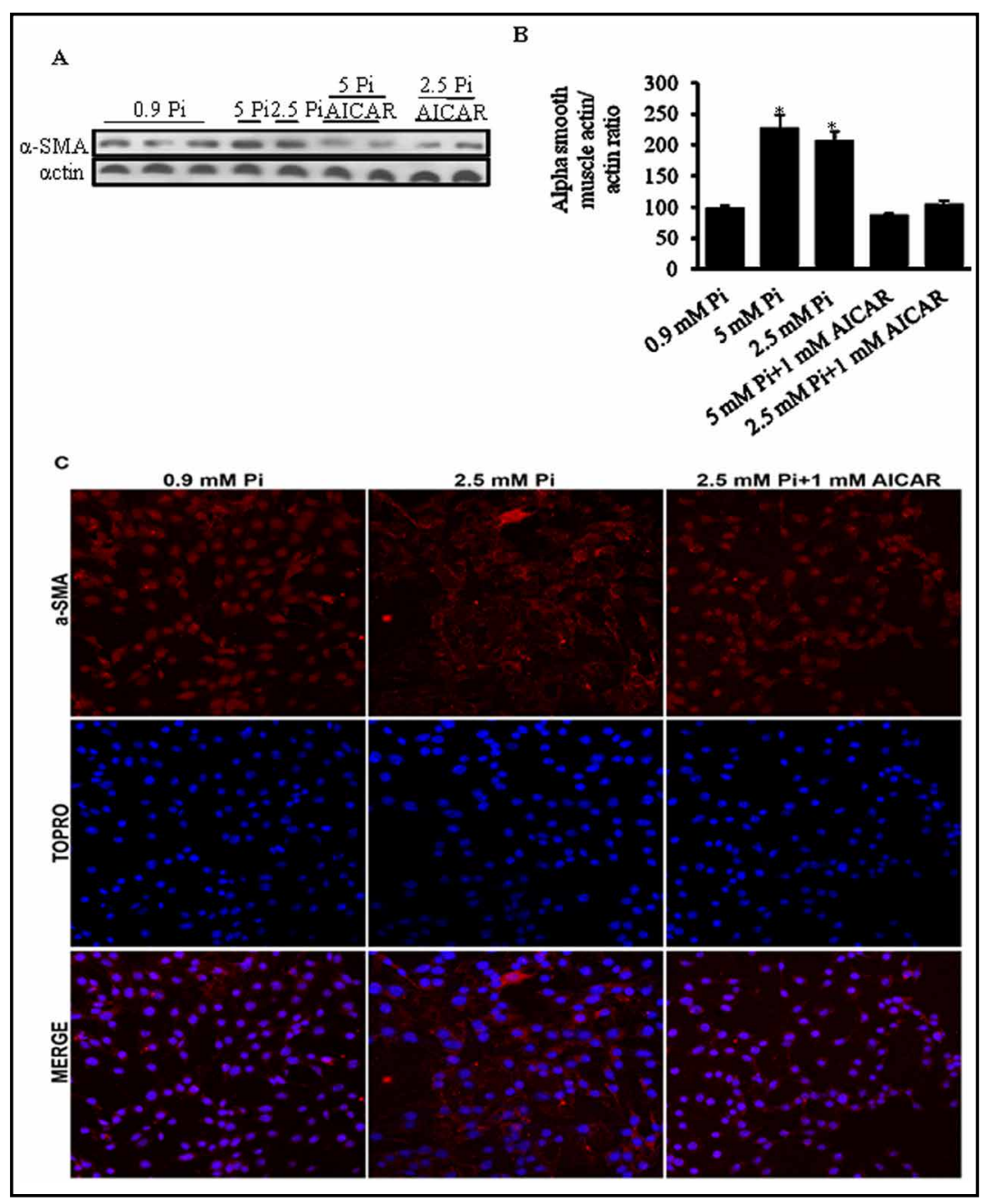

Fig. 8. High phosphate levels inactivate LKB1 in iHMCs. Representative western blot analysis of phosphorylated LKB1 (pLKB1) (Ser ${ }^{428}$ ) (a) in iHMCs under $0.9 \mathrm{mM}$ or $2.5 \mathrm{mM}$ or $5 \mathrm{mM}$ phosphate followed by quantification of pLKB1/TLKB1/actin ratio (b). The bars represent mean \pm SEM of band intensities (arbitrary densitometric units) of 3 independent experiments and $\mathrm{n}=5$ for each treatment. ${ }^{*} \mathrm{p}<0.001$ vs. $0.9 \mathrm{mM}$ phosphate.
p-LK

A
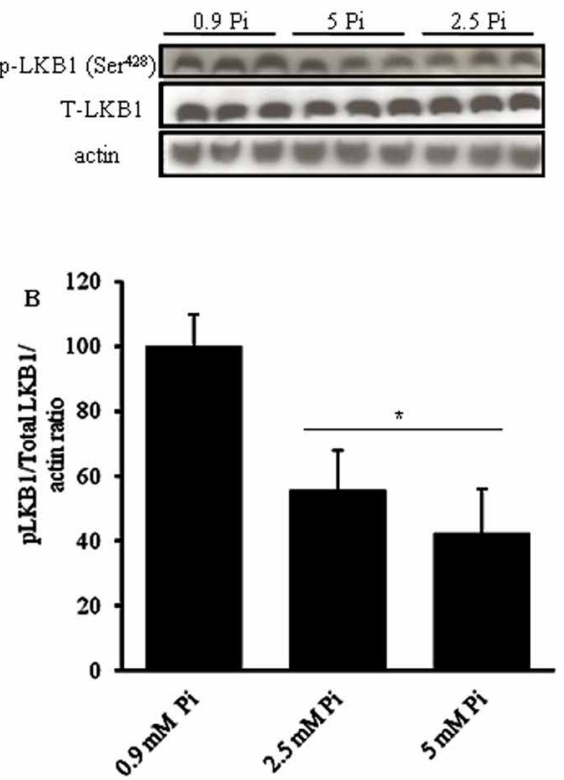
Fig. 9. High phosphate levels increase TGFß-1 signaling and ECM accumulation via NADPH oxidase activation in iHMCs. Representative western blot analysis of phosphorylated SMAD3 (a) and collagen IV expression (c) in iHMCs under $0.9 \mathrm{mM}$ or $2.5 \mathrm{mM}$ or $5 \mathrm{mM}$ phosphate alone or in combination with DPI (50 $\mathrm{nM}$ ) followed by quantification of pSMAD3/TSMAD3/actin ratio (b) and collagen IV/actin ratios (d). The bars represent mean \pm SEM of band intensities (arbitrary densitometric units) of 3 independent experiments and $n=5$ for each treatment. $* \mathrm{p}=0.015$ and $\# \mathrm{p}<0.0001$ vs. $0.9 \mathrm{mM}$ phosphate for (b). *p $=0.02$ and $\# \mathrm{p}<0.0001$ vs. 0.9 $\mathrm{mM}$ phosphate vs. $0.9 \mathrm{mM}$ phosphate for (d).

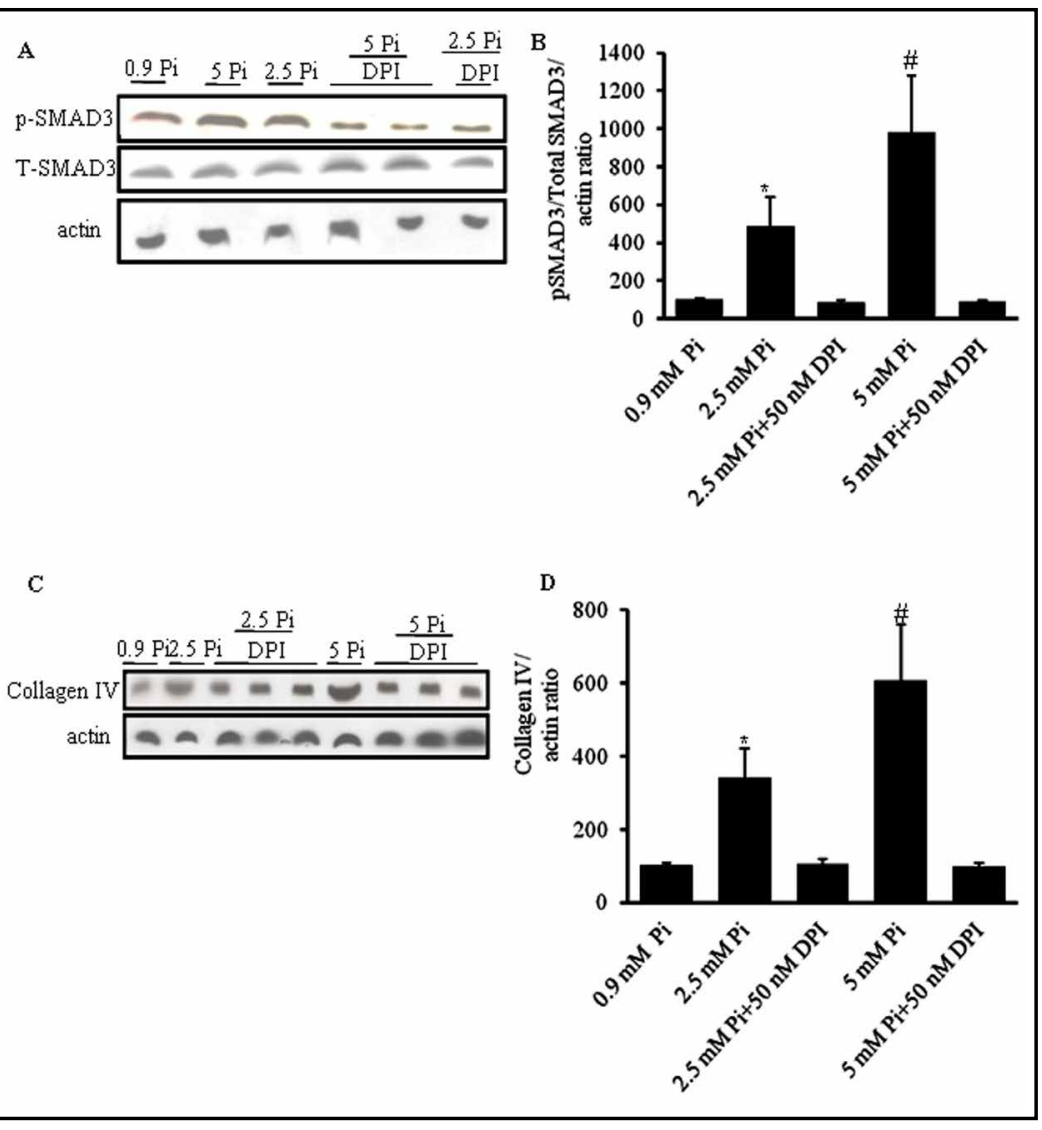

Glomerular ECM accumulation with subsequent development of glomerulosclerosis is associated with progression of CKD [7]. Maneuvers aimed at reduction in plasma Pi levels are associated with reduction of glomerulosclerosis and interstitial fibrosis in uremic rats [4]. In the current study, the medium concentrations used in mesangial cells are comparable to a 2- to 4-fold increase of serum Pi concentrations, which indicate that even mild increases in serum Pi concentrations in vivo may have a critical stimulatory role on fibrogenesis in the kidney.

Our results identify, for the first time, that NOX4 is an upstream critical activator in mediating ECM accumulation via rise in TGFß-1 signaling in mesangial cells exposed to elevated levels of $\mathrm{Pi}$, and this is in agreement with our previous study of mesangial cells exposed to diabetic conditions [11]. The upregulation of NOX4 has been linked with a rise in fibronectin $[9,10,26]$ in mesangial and tubular cells exposed, for example, to high glucose, as well as with an increase in mesangial hypertrophy under angiotensin II treatment $[27,28]$. The importance of targeting NOX4 activation as a renoprotective maneuver has been clearly demonstrated in an experimental model of diabetic nephropathy [29].

The inactivation of AMPK has been known to contribute to ECM accumulation and kidney hypertrophy $[19,30,31]$ and, more importantly, to kidney fibrosis [16]. Use of AICAR, which activates AMPK, in a model of CDK in rats led to a reduction in kidney fibrosis [17]. Our previous study [11] showed, for the first time, that the inactivation of AMPK upstream of NOX4 is a critical step in regulating both high glucose and TGFß-1-induced ECM accumulation. Our current study extends our previous findings by demonstrating that AMPK/NOX4/TGFß1 signaling also mediates high Pi-induced profibrotic damage in mesangial cells.

In conclusion, this study has identified AMPK and NOX4 as critical players in the cellular mechanism for high Pi-induced ECM accumulation via TGFß-1 signaling in human mesangial cells. The efficacy of maneuvers to increase AMPK and reduce NOX4 activity aiming to confer renal protection in vivo under hyperphosphatemia deserves further investigation. 
Papadimitriou et al.: High Phosphate Levels Increase ECM Accumulation via AMPK Inactivation in Mesangial Cells

Fig. 10. High phosphate levels increase TGFß-1 signaling via increase in NOX4 expression in iHMCs. Representative western blot analysis of NOX4 (a and c), and phosphorylated SMAD3 (e) expression in iHMCs under $0.9 \mathrm{mM}$ or $2.5 \mathrm{mM}$ or $5 \mathrm{mM}$ phosphate alone or in combination with transfection of scramble NOX4 plasmid (src $200 \mathrm{nM}$ ) or with transfection of siRNA NOX4 plasmid (si, $200 \mathrm{nM}$ ) followed by quantification of NOX4/actin ratio (b and d), and pSMAD3/TSMAD3/actin (f) ratios. The bars represent mean \pm SEM of band intensities (arbitrary densitometric units) of 3 independent experiments and $\mathrm{n}=5$ for each treatment. ${ }^{*} \mathrm{p}$ $=0.001$ vs. $0.9 \mathrm{mM}$ phosphate for (b). ${ }^{*} \mathrm{p}=0.02$ and $\# \mathrm{p}<$ 0.0001 vs. $0.9 \mathrm{mM}$ phosphate for (d). ${ }^{*} \mathrm{p}=0.002$ and \#p < 0.0001 vs. $0.9 \mathrm{mM}$ phosphate for (f).

Fig. 11. High phosphate levels increase ECM accumulation via increase in NOX4 expression in iHMCs. Representative western blot analysis of collagen IV (a), and fibronectin (c) expression in iHMCs treated as indicated followed by quantification of collagen IV/ actin (b), and fibronectin/actin (d) ratios. The bars represent mean \pm SEM of band intensities (arbitrary densitometric units) of 3 independent experiments and $n=5$ for each treatment. ${ }^{*} \mathrm{p}=0.01$ and $\# \mathrm{p}<0.0001$ vs. 0.9 $\mathrm{mM}$ phosphate for (b). ${ }^{*} \mathrm{p}$ $=0.0002$ and $\# \mathrm{p}<0.0001$ vs. $0.9 \mathrm{mM}$ phosphate for (d).
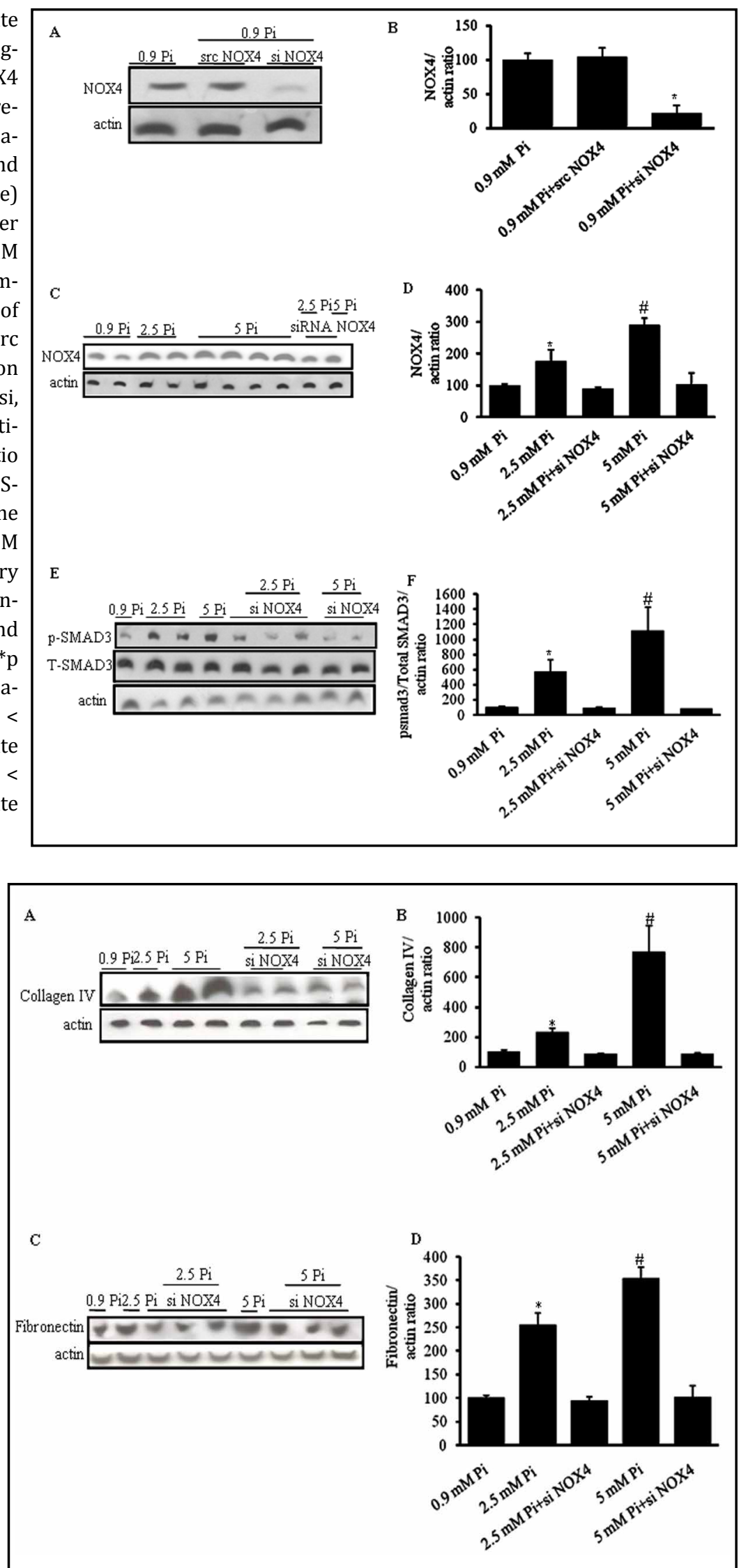
Papadimitriou et al.: High Phosphate Levels Increase ECM Accumulation via AMPK Inactivation in Mesangial Cells

Fig. 12. Schematic representation of the proposed mechanism by which elevated phosphate levels lead to ECM accumulation in mesangial cells. Abbreviations: AMPK (adenosine monophosphateactivated protein kinase), ECM (extracellular matrix accumulation), TGFß-1 (transforming growth factor $ß 1$ ), TGFßR-1 (transforming growth factor receptor $ß 1$ ).

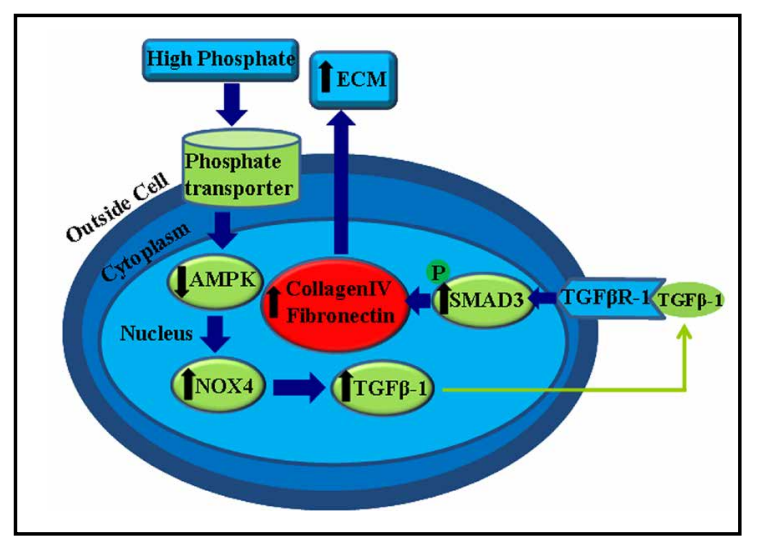

\section{Abbreviations}

AICAR (5-aminoimidazole-4-carboxamide ribonucleotide); AMPK (5' adenosine monophosphate-activated protein kinase); ANOVA (analysis of variance); $\alpha$-SMA (alpha smooth muscle actin); CKD (chronic kidney disease); DHE (Dihydroethidium); DPI (diphenyleneiodonium); DMEM (Dulbecco's modified Eagle's medium); ECM (extracellular matrix); iHMCs (immortalized human mesangial cells); LKB1 (liver kinase B1); MTT (3-(4,5-dimethylthiazol-2-yl)-2,5-diphenyltetrazolium bromide); NADPH (nicotinamide adenine dinucleotide phosphate); PFA (phosphonoformic acid); Pi (phosphate inorganic); ROS (reactive oxygen species); Scr (scramble); SEM (standard error of mean);siRNA (small interfering RNA); SO (superoxide); TGFß-1 (transforming growth factor ß-1).

\section{Acknowledgments}

The authors thank Luciana Cristina Teixeira (Department of Medicine, Nephrology Division, Federal University of São Paulo, Brazil) for helping with the culture of the iHMC line. The authors are very grateful to the personnel from the Renal Pathophysiology Laboratory, Investigation on Diabetes Complications, Faculty of Medical Sciences (FCM), Unicamp, for their invaluable help with this work.

This work was supported by the Fundação de Amparo à Pesquisa do Estado de São Paulo (FAPESP, Grant 2008/57560-0 and 2012/22452-8) and Conselho Nacional de Desenvolvimento Científico e Tecnológico (CNPq, Grant 301797/2009-9). A.P. received a postdoctoral scholarship from FAPESP.

\section{Disclosure Statement}

No conflicts of interest, financial or otherwise, are declared by the authors.

\section{References}

1 Voormolen N, Noordzij M, Grootendorst DC, Beetz I, Sijpkens Y.W, Van Manen JG, Boeschoten EW, Huisman RM, Krediet RT, Dekker FW, PREPARE study group: High plasma phosphate as a risk factor for decline in renal function and mortality in pre-dialysis patients. Nephrol Dial Transplant 2007;22:2909-2916.

72 Kestenbaum B: Phosphate metabolism in the setting of chronic kidney disease: significance and recommendations for treatment. Semin Dial 2007;20:286-294.

-3 Zoccali C, Ruggenenti P, Perna A, Leonardis D, Tripepi R, Tripepi G, Mallamaci F, Remuzzi G, REIN Study Group: Phosphate may promote CKD progression and attenuate renoprotective effect of ACE inhibition. J Am Soc Nephrol 2011;10:1923-1930. 
4 Finch JL, Lee DH, Liapis H, Ritter C, Zhang S, Suarez E, Ferder L, Slatopolsky E: Phosphate restriction significantly reduces mortality in uremic rats with established vascular calcification. Kidney Int 2013;84:1145-1153.

5 Eräranta A, Riutta A, Fan M, Koskela J, Tikkanen I, Lakkisto P, Niemelä O, Parkkinen J, Mustonen J, Pörsti I: Dietary phosphate binding and loading alter kidney angiotensin-converting enzyme mRNA and protein content in 5/6 nephrectomized rats. Am J Nephrol 2012;35:401-408.

-6 Chen Z, Chen D, McCarthy TL, Centrella M, Zhang Y, Moeckel GW: Inorganic phosphate stimulates fibronectin expression in renal fibroblasts. Cell Physiol Biochem 2012;30:151-159.

7 Chatziantoniou C, Dussaule JC: Insights into the mechanisms of renal fibrosis: is it possible to achieve regression?. Am J Physiol Renal Physiol 2005;289:F227-F234.

-8 Abboud HE: Mesangial cell biology. Exp Cell Res 2012;318:979-985.

9 Gorin Y, Block K, Hernandez J, Bhandari B, Wagner B, Barnes JL, Abboud HE: Nox4 NAD(P)H oxidase mediates hypertrophy and fibronectin expression in the diabetic kidney. J Biol Chem 2005;280:3961639626.

10 Lee DY, Wauguier F, Eid AA, Roman LJ, Choudhury GG, Khazin K, Block K, Gorin Y: Nox4 NADPH oxidase mediates peroxynitrite-dependent uncoupling of endothelial nitric oxide synthase and fibronectin expression in response to angiotensin II. Role of mitochondrial reactive oxygen species. J Biol Chem 2013;288:28668-28686.

11 Papadimitriou A, Peixoto EB, Silva KC, Lopes de Faria JM, Lopes de Faria JB: Increase in AMPK brought about by cocoa is renoprotective in experimental diabetes mellitus by reducing NOX4/TGFb-1 signalling. J Nutr Biochem 2014;25:773-784.

12 Long YC, Zierath JR: AMP-protein kinase signaling in metabolic regulation. J Clin Invest 2006;116:17761783.

13 Hallows KR, Mount PF, Pastor-Soler NM, Power DA: Role of the energy sensor AMP-activated protein kinase in renal physiology and disease. Am J Physiol Renal Physiol 2010;298:F1067-F1077.

14 Hawley SA, Davison M, Woods A, Davies SP, Beri RK, Carling D, Hardie DG: Characterization of the AMPactivated protein kinase kinase from rat liver and identification of threonine 172 as the major site at which it phosphorylates AMP-activated protein kinase. J Biol Chem 1996;271:27879-27887.

15 Hardie DG, Ross FA, Hawley SA: AMP-activated protein kinase: a target for drugs both ancient and modern. Chem Biol 2012;19:1222-1236.

16 Declèves AE, Sharma K: Novel targets of antifibrotic and anti-inflammatory treatment in CKD. Nat Rev Nephrol 2014;10:257-267.

17 Satriano J, Sharma K, Blantz RC, Deng A: Induction of AMPK activity corrects early pathophysiological alterations in the subtotal nephrectomy model of chronic kidney disease. Am J Physiol Renal Physiol 2013;305:F727-F733.

18 Dugan LL, You YH, Ali SS, Diamond-Stanic M, Miyamoto S, DeCleves AE, Andreyev A, Quach T, Ly S, Shekhtman G, Nguyen W, Chepetan A, Le TP, Wang L, Xu M, Paik KP, Fogo A, Viollet B, Murphy A, Brosius F, Naviaux RK, Sharma K: AMPK dysregulation promotes diabetes-related reduction of superoxide and mitochondrial function. J Clin Invest 2013;123:4888-4899.

19 Lee MJ, Feliers D, Mariappan MM, Sataranatarajan K, Mahimainathan L, Musi N, Foretz M, Viollet B, Weinberg JM, Choudhury GG, Kasinath BS: A role for AMP-activated protein kinase in diabetes-induced renal hypertrophy. Am J Physiol Renal Physiol 2007;292:F617-F627.

-20 Block K, Eid A, Griendling KK, Lee DY, Wittrant Y, Gorin Y: Nox4 NAD(P)H oxidase mediates Src-dependent tyrosine phosphorylation of PDK-1 in response to angiotensin II: role in mesangial cell hypertrophy and fibronectin expression. J Biol Chem 2008;283:24061-24076.

21 Banas B, Luckow B, Möller M, Klier C, Nelson PJ, Schadde E, Brigl M, Halevy D, Holthöfer H, Reinhart B, Schlöndorff D: Chemokine and chemokine receptor expression in a novel human mesangial cell line. J Am Soc Nephrol 1999;10:2314-2322.

22 Cristovam PC, Arnoni CP, De Andrade MC, Casarini DE, Pereira LG, Schor N, Boim MA: ACE-dependent and chymase-dependent angiotensin II generation in normal and glucose stimulated human mesangial cells. Exp Biol Med (Maywood) 2008;233:1035-1043.

23 Ribaldo PDB, Souza DS, Biswas SK, Block K, Lopes de Faria JM, Lopes de Faria JB : Green tea (Camelia sinensis) attenuates nephropathy by down-regulating Nox4 NADPH oxidase in diabetic spontaneously hypertensive rats. J Nutr 2009;139:96-100. 


\section{Cellular Physiology Cell Physiol Biochem 2014;34:1260-1272 and Biochemistry

Papadimitriou et al.: High Phosphate Levels Increase ECM Accumulation via AMPK Inactivation in Mesangial Cells

24 Faria AM, Papadimitriou A, Silva KC, Lopes de Faria JM, Lopes de Faria JB: Uncoupling endothelial nitric oxide synthase is ameliorated by green tea in experimental diabetes by re-establishing tetrahydrobiopterin levels. Diabetes 2012;61:1838-1847.

-25 Biswas SK, Peixoto EB, Souza DS, Lopes de Faria JB: Hypertension increases pro-oxidant and decreases antioxidant defense in the kidney in early diabetes. Am J Nephrol 2008;28:133-142.

-26 Sedeek M, Callera G, Montezano A, Gutsol A, Heitz F, Szyndralewiez C, Page P, Kennedy CR, Burns KD, Touyz RM, Hebert RL: Critical role of Nox4-based NADPH oxidase in glucose-induced oxidative stress in the kidney: implications in type 2 diabetic nephropathy. Am J Physiol Renal Physiol 2010;299:F1348-F1358.

27 Gorin Y, Ricono JM, Wagner B, Kim NH, Bhandari B, Choudhury GG, Abboud HE: Angiotensin II-induced ERK1/ERK2 activation and protein synthesis are redox-dependent in glomerular mesangial cells. Biochem J 2004;381:231-239.

28 Gorin Y, Ricono JM, Kim NH, Bhandari B, Choudhury GG, Abboud HE: Nox4 mediates angiotensin II-induced activation of Akt/protein kinase B in mesangial cells. Am J Physiol Renal Physiol 2003;285:F219-F229.

29 Jha JC, Gray SP, Barit D, Okabe J, El-Osta A, Namikoshi T, Thallas-Bonke V, Wingler K, Szyndralewiez C, Heitz F, Touyz RM, Cooper ME, Schmidt HH, Jandeleit-Dahm KA: Genetic targeting of pharmacological inhibition of NADPH oxidase Nox4 provides renoprotection in long-term diabetic nephropathy. J Am Soc Nephrol 2014;25:1237-1254.

-30 Kim MY, Lim JH, Youn HH, Hong YA, Yang KS, Park HS, Chung S, Ko SH, Shin SJ, Choi BS, Kim HW, Kim YS, Lee JH, Chang YS, CW Park: Resveratrol prevents renal lipotoxicity and inhibits mesangial cell glucotoxicity in a manner dependent on the AMPK-Sirt1-PGC1a axis in db/db mice. Diabetologia 2013;56:204-217.

-31 Ding DF, You N, Wu XM, Xu JR, Hu AP, Ye XL, Zhu Q, Jiang XQ, Miao H, Liu C, Lu YB: Resveratrol attenuates renal hypertrophy in early-stage diabetes by activating AMPK. Am J Nephrol 2010;31:363-374. 